\title{
Triggering receptor expressed on myeloid cells: role in the diagnosis of lung infections
}

\author{
L. Richeldi*, M. Mariani\# ${ }^{\#}$ M. Losi*, F. Maselli*, L. Corbetta*, C. Buonsanti" , M. Colonna ${ }^{\Uparrow}$, F. Sinigaglia", \\ P. Panina-Bordignon ${ }^{\#}$, L.M. Fabbri*
}

Triggering receptor expressed on myeloid cells: role in the diagnosis of lung infections. L. Richeldi, M. Mariani, M. Losi, F. Maselli, L. Corbetta, C. Buonsanti, M. Colonna, F. Sinigaglia, P. Panina-Bordignon, L. M. Fabbri. (C) ERS Journals Ltd 2004.

ABSTRACT: The triggering receptor expressed on myeloid cells (TREM)-1 is a recently described molecule, which plays an important role in myeloid cell-activated inflammatory responses. TREM-1 is expressed on blood neutrophils and monocytes, and also on alveolar macrophages, thus suggesting a potential role in lung inflammatory responses against infections.

To investigate the differential expression of TREM-1 in lung infections, its levels were assessed in bronchoalveolar lavage specimens from patients with communityacquired pneumonia or tuberculosis. TREM-1 was also investigated in patients with interstitial lung diseases, as a model of noninfectious inflammatory disease of the lung.

TREM-1 expression was significantly increased in lung neutrophils and in lung macrophages of patients with pneumonia $(n=7 ; 387.9 \pm 61.4$ and $660.5 \pm 18.3$, respectively) compared with patients with pulmonary tuberculosis $(n=7 ; 59.2 \pm 13.1$ and 80.6 \pm 291.2$)$ and patients with interstitial lung diseases $(n=10 ; 91.8 \pm 23.3$ and 123.9 \pm 22.8$)$. In contrast, TREM-1 expression on peripheral blood neutrophils was no different among the three groups.

In conclusion, these data suggest that triggering receptor expressed on myeloid cells-1 is selectively expressed in the lungs of patients with pneumonia caused by extracellular bacteria and not in patients with tuberculosis, providing a potential marker for differential diagnosis.

Eur Respir J 2004; 24: 247-250.
*Section of Respiratory Diseases, Dept of Oncology and Haematology, University of Modena and Reggio Emilia, Modena, and ${ }^{\#}$ BioXell SpA, Milan, Italy. "Dept of Pathology and Immunology, Washington University School of Medicine, St Louis, MO, USA.

Correspondence: L.M. Fabbri, Section of Respiratory Diseases, Dept of Oncology and Haematology, University of Modena and Reggio Emilia, Via del Pozzo 71 - 41100 Modena, Italy.

Fax: 39594224231

E-mail: fabbri.leonardo@unimo.it

Keywords: Bacterial infections

bronchoalveolar lavage

neutrophils

pneumonia

triggering receptor expressed on myeloid cells- 1 tuberculosis

Received: January 42004

Accepted after revision: March 302004
The immune response to pathogens relies on both innate and adaptive immunity. The cells of the innate immune system provide a first line of host defence, reacting rapidly to external stimuli, including various pathogens and transformed or infected cells [1]. The innate response is mediated largely by white blood cells, specifically neutrophils and macrophages. These cells can phagocytose and kill pathogens, and can induce a host immune response by releasing several inflammatory mediators and cytokines [2, 3].

In the lung, the local inflammatory response to the external pathogens is the primary mechanism of defence against infections. Innate immunity in the lung is mediated by neutrophils, monocytes and alveolar macrophages [2]. Myeloid cells express several activating receptors belonging to the immunoglobulin (Ig) superfamily, e.g. the human leukocyte Ig-like receptors [4], the signal regulatory proteins [4] and the triggering receptors expressed on myeloid cells (TREMs) [4].

Human TREM-1 is a $30-\mathrm{kDa}$ glycoprotein of the immunoglobulin superfamily which, together with TREM-2 [5], constitutes the prototypic member of the TREM family, identified on human chromosome 6 and expressed on myeloid cells $[4,6]$. TREM-1 expression has been found on neutrophils and monocytes, and may contribute to the maturation of stem cells to monocytes [7]. Upon cross-linking, TREM-1 induces the secretion of several pro-inflammatory cytokines and chemokines, such as tumour necrosis factor (TNF)- $\alpha$, interleukin (IL)-8 and monocyte chemoattractant protein-1.
In addition, TREM-1 triggers degranulation of neutrophils, $\mathrm{Ca}^{2+}$ mobilisation and tyrosine phosphorylation of several proteins, in particular mitogen activated proteins kinases ERK1 and ERK2. The cellular expression of TREM-1 is up-regulated following Toll-like receptor activation, with an accompanying increased production of the pro-inflammatory cytokines TNF- $\alpha$ and granulocyte-macrophage colony-stimulating factor, while the production of the anti-inflammatory cytokine IL-10 is reduced. This pattern of activation has suggested a role for this receptor in acute inflammation [8]. Moreover, TREM-1 is also expressed on lung alveolar macrophages, and this tissue-specific pattern of expression has suggested a potential role for this molecule in the activation of the local inflammatory response during lung infections [6].

In vitro studies have shown that the expression of TREM-1 is strongly up-regulated by extracellular bacteria, as well as by their cell wall components, and by fungi, but not by mycobacteria [4, 8]. In vivo, the expression of TREM-1 is strongly up-regulated on neutrophils infiltrating skin lesions in impetigo (caused by Staphylococcus aureus) or in granulomatous lymphadenitis caused by extracellular bacteria or fungi $[4,9]$. In these lesions, TREM-1 is expressed not only in infiltrating neutrophils, but also in the epithelioid cells of monocytic origins that surround the granulomatous reaction [6]. In contrast, TREM-1 is poorly expressed in noninfectious inflammatory diseases such as psoriasis, ulcerative colitis and vasculitis [4, 9], and in granulomatous infections caused by 
Table 1.-Characteristics of study subjects in the three disease groups

\begin{tabular}{lccc}
\hline Characteristics & Group A & Group B & Group C \\
\hline Subjects n & 7 & 7 & 10 \\
Male n (\%) & $4(57)$ & $4(57)$ & $6(60)$ \\
Age yrs mean & 61.4 & 40.9 & 49.7 \\
White n (\%) & $7(100)$ & $3(43)$ & $9(90)$ \\
\hline
\end{tabular}

Group A: patients with community-acquired pneumonia likely to be caused by extracellular bacteria; group B: patients with pulmonary tuberculosis; group C: patients with noninfectious interstitial lung diseases.

Mycobacterium tuberculosis or in foreign body granulomas [6]. Recently, the levels of TREM-1 in bronchoalveolar lavage (BAL) samples of patients receiving mechanical ventilation have been shown to be the strongest independent predictor of ventilator-associated pneumonia in a logistic regression analysis [10].

To investigate the role of TREM-1 ex vivo in different lung infections, its expression on alveolar macrophages and neutrophils from BAL specimens was assessed, in addition to its expression on peripheral blood neutrophils of patients with community-acquired pneumonia (CAP), pulmonary tuberculosis (TB) or noninfectious interstitial lung diseases (ILD).

\section{Methods}

\section{Study population}

Between November 1, 2000, and May 31, 2002, 66 patients were prospectively enrolled in the study on the basis of a clinical indication to perform a bronchoscopy with BAL for the diagnosis of lung infection or a diffuse interstitial lung disorder. The study was approved by the Ethical Committee of the University of Modena and Reggio Emilia (Modena, Italy) and written informed consent was obtained from all patients enrolled. A number of patients were excluded for the following reasons: 24 due to the low number of viable cells in BAL specimens; 12 due to the lack of complete clinical followup data; two due to the presence of massive amounts of blood in the BAL specimens; and four because of previous treatment with nonspecified broad-spectrum antibiotics. Twentyfour patients completed the study protocol: 14 (58.3\%) were male, $19(79.2 \%)$ were White and they had a median age of 50.5 yrs (table 1). On the basis of clinical, radiographical and microbiological data, patients were assigned to one of three groups (table 2). Group A $(n=7)$ consisted of patients with a diagnosis of CAP likely to be caused by extracellular bacteria (CAPEB): three patients had microbiological evidence of a pulmonary infection, based upon a positive culture from BAL samples (Haemophilus parainfluenzae, Pseudomonas aeruginosa and $S$. pneumoniae); and four patients were diagnosed with CAP on the basis of a typical clinical and/or radiological presentation, and the rapid response to antibiotic therapy (including $\beta$-lactam, $\beta$-lactam and $\beta$-lactamase inhibitors, and 2nd and 3rd generation cefalosporins). Patients receiving macrolides and/or fluoroquinolones were excluded from the study, and an aetiology of the CAPEB due to "atypical" intracellular bacteria (Mycoplasma pneumoniae, Chlamydia pneumoniae and Legionella pneumophila) was ruled out in all patients, based upon negative serological test results on both acute and convalescent serum samples. Group B $(n=7)$ consisted of patients with pulmonary TB: two patients were diagnosed on the basis of microscopic and/or culture results positive for M. tuberculosis; and in five patients the diagnosis was made at the 3-month follow-up on the basis of the clinical and radiological response to a complete course of anti-TB therapy $[4,11]$. In all patients in both groups A and B, BAL was performed before initiation of any antibiotic therapy. Group $C(n=10)$ consisted of patients with ILD and without any evidence of lung infection on BAL samples. This group was chosen as a "control diseased group", in order to test the

Table 2.-Groups to which study subjects were assigned

\begin{tabular}{|c|c|c|c|}
\hline Group & Diagnosis & Chest radiographical appearance & Site of BAL \\
\hline \multirow[t]{7}{*}{ A } & CAPEB & Basilar left opacity & Lingula $\left(\mathrm{B}_{5}\right)$ \\
\hline & CAPEB & Lower left lobe opacity & Lower left lobe $\left(\mathrm{B}_{6}\right)$ \\
\hline & CAPEB & Bilateral reticulonodular pattern & Lower right lobe $\left(\mathrm{B}_{9}\right)$ \\
\hline & CAPEB & Lower right lobe opacity & Upper right lobe $\left(\mathrm{B}_{2}\right)$ \\
\hline & CAPEB & Upper right lobe opacity & Middle lobe \\
\hline & CAPEB & Middle lobe opacity & Middle lobe \\
\hline & CAPEB & Middle lobe volume loss & Middle lobe \\
\hline \multirow[t]{7}{*}{ B } & TB & Lower right posterior opacity & Lower right lobe $\left(\mathrm{B}_{10}\right)$ \\
\hline & TB & Diffuse left multiple opacities & Upper left lobe $\left(\mathrm{B}_{3}\right)$ \\
\hline & $\mathrm{TB}$ & Left opacity & Middle lobe \\
\hline & TB & Middle and upper lobe infiltrates & Middle lobe \\
\hline & TB & Miliary pattern & Lower right lobe $\left(\mathrm{B}_{6}\right)$ \\
\hline & TB & Widespread right opacities & Upper right lobe $\left(\mathrm{B}_{1}\right)$ \\
\hline & TB & Upper left opacity & Upper left lobe $\left(\mathrm{B}_{3}\right)$ \\
\hline \multirow[t]{10}{*}{$\mathrm{C}$} & Histiocytosis X & Bilateral diffuse reticular pattern & Middle lobe \\
\hline & Sarcoidosis & Bilateral diffuse opacities & Middle lobe \\
\hline & Hypersensitivity pneumonia & Basilar interstitial infiltrates & Lingula \\
\hline & Pneumoconiosis & Bilateral nodular pattern & Middle lobe \\
\hline & Eosinophilic pneumonia & Bilateral upper middle opacity & Upper left lobe \\
\hline & Hypersensitivity pneumonia & Upper right opacity & Upper right lobe \\
\hline & Histiocytosis X & Bilateral interstitial infiltrates & Middle lobe \\
\hline & Rheumatoid lung & Diffuse interstitial infiltrates & Lower left lobe $\left(\mathrm{B}_{6}\right)$ \\
\hline & Alveolar proteinosis & Bilateral diffuse opacities & Middle lobe \\
\hline & Idiopathic pulmonary fibrosis & Interstitial pattern & Middle lobe \\
\hline
\end{tabular}

BAL: bronchoalveolar lavage; CAPEB: community-acquired pneumonia likely to be caused by extracellular bacteria; TB: tuberculosis. See Methods section for a description of subjects in each group. 
hypothesis of a preferential expression of TREM-1 in pneumonia due to extracellular bacteria, compared to inflammatory lung disorders not primarily due to an infectious cause.

\section{Bronchoalveolar lavage and cytofluorimetric analysis}

BAL was performed using a total of $150 \mathrm{~mL}$ of sterile physiological saline solution in three consecutive $50-\mathrm{mL}$ aliquots. The recovered fluid was filtered through a single layer of gauze to remove mucus and divided into two aliquots: a 10-mL aliquot was used for TREM-1 analysis (see below) and the remaining sample was used for differential cell count. For the flow cytometric analysis, cells in BAL specimens were stained with either an anti-TREM-1 monoclonal antibody (21C7), as previously described [4], or with a control purified mouse $\operatorname{IgG} 1_{\mathrm{K}}$ monoclonal immunoglobulin isotype antibody (33811A; PharMingen International, Becton Dickinson Biosciences, San Diego, CA, USA). Double staining was performed using fluorescein isothiocyanate-conjugated mouse anti-human CD14 or mouse anti-human CD15 monoclonal antibody (PharMingen International) to detect monocyte/ macrophages and granulocytes, respectively. Granulocytes were purified from peripheral blood according to standard procedures [4] and stained as described for cells obtained by BAL.

Based upon dot-plot data, TREM-1 levels were measured both as expression per cell (therefore correcting for the total number of different cellular types) and as mean fluorescence (MF) divided by total number of cells as measured in differential cell counts. Data are presented as MF \pm SEM.

\section{Results}

Cellular profiles of BAL fluids in the three different groups were not statistically different, even if, as expected, there was a trend for a difference in neutrophils among patients with CAPEB (\% neutrophils of BAL cells: group A 65.1 19.4 ; group B 21.5 \pm 9.8 ; group C $48.0 \pm 10.1$ ) and in lymphocytes among patients with TB ( $\%$ lymphocytes of BAL cells: group A 28.0 \pm 15.3 ; group B 39.5 \pm 20.0 ; group C 15.1 \pm 14.0 ; $p=N S$ for all comparisons).

Flow cytometry of BAL fluids showed that TREM-1 was selectively expressed on lung neutrophils and macrophages, but was not expressed on lymphocytes, thus confirming previously published data. TREM-1 expression was significantly higher in cells of patients from group A (MF $246.1 \pm 77.1)$, compared to patients from group B (MF $38.1 \pm 7.9, \mathrm{p}<0.05)$ or group $\mathrm{C}(\mathrm{MF} 41.9 \pm 8.1, \mathrm{p}<0.001)$ (fig. 1). Double staining experiments performed on a subgroup of patients (five out of seven for group A, seven out of seven for group B and nine out of 10 for group C) revealed that the differences in TREM-1 expression were due to an increase in the expression on both neutrophils (group A $387.9 \pm 61.4$, group B 59.2 $\pm 13.1, \mathrm{p}<0.001$ versus group $\mathrm{A}$; group $\mathrm{C} 91.8 \pm 23.3, \mathrm{p}<0.001$ versus group $\mathrm{A})$ and macrophages (group A $660.5 \pm 18.3$, group B 80.6 $\pm 291.2, \mathrm{p}<0.05$ versus group $\mathrm{A}$; group $\mathrm{C} 123.9 \pm 22.8, \mathrm{p}<0.05$ versus group $\mathrm{A}$ ).

The expression of TREM-1 in peripheral blood neutrophils was similar in the three groups, both at baseline (group A

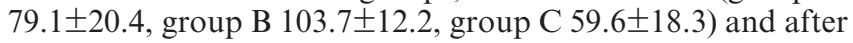
in vitro stimulation of blood cells with lipopolysaccharide (group A 115.1 \pm 61.8 , group B 139.6 \pm 23.6 , group C

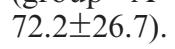

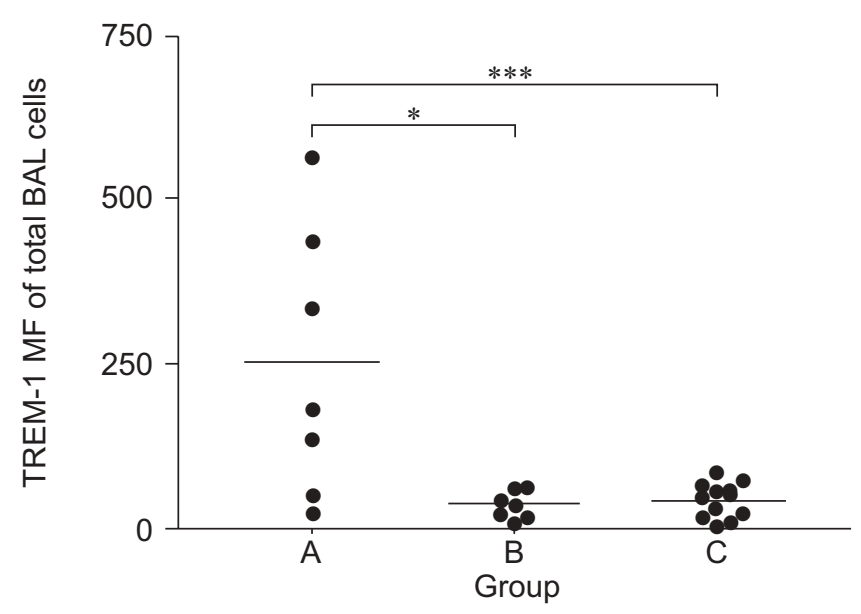

Fig. 1.-Triggering receptor expressed on myeloid cells (TREM)-1 expression on total bronchoalveolar (BAL) cells is significantly higher in patients with community-acquired pneumonia likely to be caused by extracellular bacteria (group A) than in patients with pulmonary tuberculosis (group B; *: $\mathrm{p}<0.05$ ) or patients with noninfectious interstitial lung diseases (group $\mathrm{C} ; * * *$ : $\mathrm{p}<0.001$ ). Data are presented as TREM-1 mean fluorescence (MF) of total BAL cells, subtracted from isotype control.

\section{Discussion}

In this study, the ex vivo expression of the new TREM-1 receptor on lung cells obtained from patients with CAPEB, TB or other inflammatory lung diseases was examined. TREM-1 was found to be selectively expressed on lung neutrophils and macrophages but not on lung lymphocytes, and its expression was markedly increased on lung neutrophils and macrophages from patients with CAPEB but not from patients with pulmonary TB or ILD.

These data confirm what has previously been shown in vitro, i.e. the different ability of extracellular and intracellular pathogens to induce TREM-1 expression [4]. Indeed, only Gram-positive, Gram-negative or bacterial components (i.e. lipopolysaccharide or lipoteichoic acid), but not intracellular bacteria such as $M$. tuberculosis or components of its cell wall (mycolic acid), are able to induce TREM-1 upregulation on neutrophils in vitro [4]. Moreover, in skin lesions caused by various pathogens, TREM-1 is expressed only in the presence of extracellular bacteria or fungi, and it is not detected on neutrophils. A similar pattern of TREM-1 expression was observed in BAL specimens: TREM-1 was highly expressed on cells of patients with pneumonia due to extracellular bacteria, but not on cells of patients with active TB or non-infectious ILD. Conversely, TREM-1 was found to be comparably expressed on peripheral blood neutrophils in all subjects, suggesting that the TREM-1-stimulating pathway is activated locally in the lung parenchyma during infection. The functional features of TREM-1 observed in the human lung suggest that it may have a key role in acute lung infectious inflammatory diseases caused by extracellular bacteria, in which the inflammatory response is mediated by neutrophils and alveolar macrophages, as it is in pneumonia but not in TB. This could imply that different mechanisms are involved in the activation of neutrophils and amplification of the inflammatory response in these two types of infections. Moreover, different mechanisms of TREM-1 regulation are possibly implicated.

Pneumonia is an acute infection of the lung parenchyma that is caused by pathogens, including various bacterial species, fungi, viruses and parasites, and is characterised by recruitment of phagocytic cells, in particular alveolar macrophages 
and neutrophils. These cells act synergistically to generate an acute inflammatory response and thereby eliminate the pathogens by phagocytosis, leading to complete resolution of the infection. Conversely, the lack of expression of TREM-1 on inflammatory cells during TB infection may allow M. tuberculosis to elude microbicidal mechanisms. In response to mycobacterial infection, the accumulation of activated macrophages and lymphocytes at the site of infection produces granulomatous lesions capable of limiting mycobacterial growth. However, mycobacteria can survive within the macrophages of the infected host, inducing a persistent infection. In this case, the agent causing the inflammatory process is not removed and the infection progresses to a chronic stage. Thus, one could speculate that during acute bacterial pneumonia, TREM-1 and microbial products may synergise by amplifying the inflammatory responses via different pathways. In this manner, in acute human lung infections, TREM-1 may represent a mechanism by which the innate immune system reacts to the presence of different infectious agents (e.g. extracellular versus intracellular bacteria), thus contributing to the clearance of the extracellular bacteria. In this context, TREM-1 expression on the lung-infiltrating inflammatory cells could be a useful diagnostic marker and its expression merits to be analysed in the future in other types of patients with infectious pulmonary processes, such as those with "atypical" pneumonia and with nosocomial pneumonia.

It should be noted that, even if the results presented here are statistically significant, the study population has been carefully selected to compare patients with acute pneumonia due to extracellular pathogens with patients affected by pulmonary inflammation due to the intracellular pathogen, M. tuberculosis. These two groups represent two distant parts of a wide spectrum of pulmonary infections, and lung expression of TREM-1 has not been measured in patients with pneumonia due to "atypical" intracellular pathogens or to fungi. Moreover, two patients with CAPEB showed lung expression of TREM-1 at low levels: one of them was the only one receiving antibiotic therapy at the time of BAL and, even though clinical and radiographical data confirmed the diagnosis of CAPEB, no germs were isolated from the BAL fluid, and the hypothesis that antibiotics interfered with TREM-1 expression cannot be ruled out; the second patient with low TREM-1 expression had bilateral pulmonary infiltrates and, even though $H$. parainfluenzae was isolated from BAL, his clinical course was subacute, and once again the possibility that TREM-1 expression may vary in different forms or stages of CAPEB cannot be excluded. However, the data presented here are in agreement with those recently described in patients with CAP and ventilator-associated pneumonia [10]. The recent identification of the unique dimeric structure of the TREM-1 molecule [12] makes the identification of the ligand(s) of TREM-1, and therefore its modulation in vivo, more likely. This approach could lend further support to the treatment of patients with severe bacterial pneumonia associated with the clinical presentation of the acute respiratory distress syndrome, in which a downregulation of the acute inflammatory process in the lung could be potentially beneficial.

In 2000 in the UK, only $60 \%$ of cases of pulmonary tuberculosis have been culture-confirmed [13]. Therefore, in a significant fraction of tuberculosis cases, diagnosis still relies on clinical, radiological or ex juvantibus criteria alone. Furthermore, both diagnosis and treatment of tuberculosis are often delayed, even in the inpatient hospital setting [14]. In this context, the rapid quantification of the levels of triggering receptor expressed on myeloid cells-1 in bronchoalveolar lavage samples could represent a further tool in refining the diagnostic spectrum in patients presenting with undefined pulmonary infiltrates.

\footnotetext{
Acknowledgements. The authors would like to thank M. McKenney for help in editing the manuscript and E. Veratelli for expert secretarial support.
}

\section{References}

1. Janeway CA Jr, Medzhitov R. Innate immune recognition. Annu Rev Immunol 2002; 20: 197-216.

2. Zhang P, Summer WR, Bagby GJ, Nelson S. Innate immunity and pulmonary host defense. Immunol Rev 2000; 173: 39-51.

3. Aderem A, Ulevitch RJ. Toll-like receptors in the induction of the innate immune response. Nature 2000; 406: 782-787.

4. Bouchon A, Dietrich J, Colonna M. Cutting edge: inflammatory responses can be triggered by TREM-1, a novel receptor expressed on neutrophils and monocytes. J Immunol 2000; 164: 4991-4995.

5. Bouchon A, Hernandez-Munain C, Cella M, Colonna M. A DAP12-mediated pathway regulates expression of $\mathrm{CC}$ chemokine receptor 7 and maturation of human dendritic cells. J Exp Med 2001; 194: 1111-1122.

6. Colonna M, Facchetti F. TREM-1 (triggering receptor expressed on myeloid cells): a new player in acute inflammatory responses. J Infect Dis 2003; 187: Suppl. 2, S397S401.

7. Gingras MC, Lapillonne H, Margolin JF. TREM-1, MDL1 , and DAP12 expression is associated with a mature stage of myeloid development. Mol Immunol 2002; 38: 817-824.

8. Bleharski JR, Kiessler V, Buonsanti C, et al. A role for triggering receptor expressed on myeloid cells-1 in host defense during the early-induced and adaptive phases of the immune response. J Immunol 2003; 170: 3812-3818.

9. Bouchon A, Facchetti F, Weigand MA, Colonna M. TREM-1 amplifies inflammation and is a crucial mediator of septic shock. Nature 2001; 410: 1103-1107.

10. Gibot S, Cravoisy A, Levy B, Bene MC, Faure G, Bollaert PE. Soluble triggering receptor expressed on myeloid cells and the diagnosis of pneumonia. N Engl J Med 2004; 350: $451-458$.

11. Diagnostic standards and classification of tuberculosis in adults and children. Am J Respir Crit Care Med 2000; 161: 1376-1395.

12. Radaev S, Kattah M, Rostro B, Colonna M, Sun PD. Crystal structure of the human myeloid cell activating receptor TREM-1. Structure (Camb) 2003; 11: 1527-1535.

13. Annual report on tuberculosis cases reported in 2000 in England, Wales and Northern Ireland. London, Tuberculosis Section Communicable Disease Surveillance Centre, 2003.

14. Rao VK, Iademarco EP, Fraser VJ, Kollef MF. Delays in the suspicion and treatment of tuberculosis among hospitalized patients. Ann Intern Med 1999; 130: 404411. 